\title{
Germanica
}

\section{Métaphysique et Rationalisme. Le débat littéraire aux Pays-Bas entre 1916 et 1950}

Metafysiek en rationalisme. Het literaire debat in Nederland tussen 1916 en 1950

\section{J.-J. Oversteegen}

Traducteur : Nicolle Dhaynaut

\section{OpenEdition Journals}

Édition électronique

URL : http://journals.openedition.org/germanica/1936

DOI : 10.4000/germanica.1936

ISSN : 2107-0784

Éditeur

Université de Lille

\section{Édition imprimée}

Date de publication : 31 décembre 1996

Pagination : 15-32

ISSN : 0984-2632

\section{Référence électronique}

J.-J. Oversteegen, « Métaphysique et Rationalisme. Le débat littéraire aux Pays-Bas entre 1916 et $1950 »$, Germanica [En ligne], 19 | 1996, mis en ligne le 04 juin 2013, consulté le 06 octobre 2020. URL http://journals.openedition.org/germanica/1936 ; DOI : https://doi.org/10.4000/germanica.1936

Ce document a été généré automatiquement le 6 octobre 2020.

(c) Tous droits réservés 


\title{
Métaphysique et Rationalisme. Le débat littéraire aux Pays-Bas entre 1916 et 1950
}

\author{
Metafysiek en rationalisme. Het literaire debat in Nederland tussen 1916 en 1950
}

\author{
J.-J. Oversteegen \\ Traduction : Nicolle Dhaynaut
}

1 On ne peut nier que, contrairement à la situation en Flandre, le débat littéraire ne se soit concentré aux Pays-Bas dans un cercle très restreint hors des contacts avec les autres pays d'Europe. Ce provincialisme n'est sans doute pas dénué de charme, celui de la pureté pourrait-on dire, mais cela ne serait pas un motif suffisant pour s'y arrêter plus longuement. Il existe un second avantage à cet isolement néerlandais : la nonbelligérance qui règne aussi dans le domaine littéraire. Je ne pense même pas spécialement à l'ouverture aux activités supranationales plus ou moins neutres telles que celles de la revue De Stijl de Théo van Doesburg (1883-1931) qui atteindra plus tard la célébrité, car celles-ci étaient étrangères aux polémiques des coryphées littéraires des années vingt et trente. Je pense plutôt au fait que certaines questions restent plus longtemps en suspens dans une situation isolée comme celle-ci, la vie littéraire y étant beaucoup plus lente qu'à l'extérieur de sorte que les motifs y deviennent visibles alors qu'ailleurs, tels des palimpsestes, ils disparaissent facilement sous d'autres éléments. Ceci vaut par exemple pour la permanence de l'effet de l'opposition du XIX ${ }^{\mathrm{e}}$ entre «l'art pour l'art » et l'engagement social qui donne d'une part, dans la « réserve » que constituent les Pays-Bas, des relations transversales avec l'opposition traditionnelle entre catholicisme et protestantisme et d'autre part avec la toute aussi ancienne polarité européenne rationalisme/romantisme, "métaphysique » («transcendant»), «temporel» (réaliste). Les groupes qui se sont formés entre les deux guerres dans le cadre de la vie littéraire néerlandaise autour de ces oppositions seront l'objet de mon intérêt ainsi que la manière avec laquelle, à la fin de cette période, dans les années 1940 à 1950, on a commencé à tendre à une synthèse dont le point de départ n'était pas le choix conduisant à une alternative radicale mais ce qui caractérise les productions 
littéraires de valeur, aspects qui, quels que contradictoires qu'ils puissent être, ne peuvent se passer les uns des autres. Dans une langue difficile à comprendre hors des frontières des Pays-Bas (les traducteurs éprouvent en effet toujours de grandes difficultés à transposer cette terminologie) on parle de caractéristiques de la littérature qui ne sont certes pas exclusivement propres à la situation néerlandaise. Si cela n'était pas le cas, je ferais mieux de me taire, mais les particularités du dialogue littéraire néerlandais me permettent de mettre en évidence des liens verticaux montrant que le débat littéraire du $\mathrm{XX}^{\mathrm{e}}$ siècle comporte un élément historique important. Cet élément est ailleurs bien souvent moins perceptible, non pas parce que les littérateurs néerlandais auraient été plus conscients que dans les autres pays des liens historiques mais plutôt justement parce que la conscience historique est peu développée au PaysBas, si bien que l'on ne réalise que rarement qu'il n'y a rien de nouveau sous le soleil. Une certaine innocence donc, qui fait que les participants à ce dialogue réactualisent sans en prendre conscience d'anciens points de vue considérés dans d'autres pays comme définitivement obsolètes et donc indignes d'une nouvelle attention tandis qu'en réalité ils continuent de jouer un rôle sous-jacent.

2 Le débat littéraire n'est pas seul à présenter cette continuité de points de vues "datés ", la littérature, elle-même produit bien souvent, sans que cela provienne de quelque programme, un amalgame de composantes qui ont ailleurs été séparées par le temps. J. Slauerhoff, par exemple (Louis Fessard lui a consacré une thèse en France) allie la sensibilité romantique allemande et française du début du XIXe siècle à une présentation moderniste, non pas sur la base d'une stratégie littéraire bien définie mais parce que dans la formation littéraire de Slauerhoff ces composantes sont inséparables. On constate l'isolement du jardin hollandais en considérant la situation et les idées des premiers novateurs: Théo van Doesburg, déjà nommé, (dont l'œuvre poétique est signée I.-K. Bonset) et Herman van den Bergh (1897-1967). Le premier, et ceci vient également d'être dit, aurait pu être à l'origine d'un lien avec les mouvements européens d'avant-garde, tels que le cubisme, le constructivisme, le futurisme et le dadaïsme mais ceci ne réussit que dans le domaine des arts plastiques et de l'architecture (Mondriaan, Bart van der Leck, Rietveld, Oud). D'un point de vue littéraire, l'impact de la revue littéraire de Van Doesburg, De Stijl ne fut que marginal et passager. Herman van den Bergh qui depuis 1917 commençait à dominer la revue Het Getij créée l'année précédente fut le novateur néerlandais le plus marquant et le plus influent des années qui suivirent la première guerre mondiale. Le plus jeune d'entre eux, Hendrik Marsman, expressionniste et vitaliste né en 1899, est au départ tellement lié à Van den Bergh qu'il reprend en partie les métaphores et le vocabulaire même de celui-ci. Aussi profonde qu'ait été l'admiration de Marsman, surtout après 1921, pour l'expressionnisme allemand il n'est jamais pour citer le plus éminent des artistes d'avant-garde néerlandais, le flamand Paul van Ostaijen, que pour «50\% expressionniste ». Ceci est dû en premier lieu au fait que le radicalisme qui caractérise les mouvements internationaux et que l'on retrouve également chez van Ostaijen n'a eu que peu de succès aux Pays-Bas, même pas chez le passionné et parfois un peu autoritaire Marsman. Qu'il soit redevable à Herman van den Bergh du principe de ses nouvelles conceptions est déjà en soi plutôt une preuve de modération que de fanatisme et met en évidence une relation avec la situation littéraire locale et non avec la situation internationale. Est-ce là une question de nature d'un peuple ayant tendance à éviter les extrêmes, ce qui mène sans doute à la tolérance mais aussi à la prudence 
civile ? Ceci est bien possible. Mais ce genre de généralisations est difficile à prouver. Il est donc préférable de s'en tenir aux faits (littéraires).

3 La poésie de Herman van den Bergh s'oppose par sa conception païenne à celle de ses prédécesseurs mais d'un point de vue formel - dans le cadre de l'avant-garde internationale ceci est un argument déterminant - on ne peut que difficilement le qualifier de novateur. Il pratique le "vers libre», rien donc de révolutionnaire et s'oppose dans ses essais sur la poésie au «culte du mot », ce que n'a pas précisément fait l'avant-garde internationale. Dans ces cercles on n'aurait sans doute même pas compris ce que Van den Bergh voulait bien dire avec ses diatribes contre le mot isolé, pour cela il fallait être nourri dans le sérail des lettres néerlandaises. Ce contre quoi Van den Bergh se révoltait, c'était les idées encore influentes des poètes impressionnistes de la fin du siècle précédent : quand il oppose la "phrase " au " mot » il ne pense absolument pas à l'indépendance du signe linguistique considéré comme image typographique comme chez les dadaïstes par exemple (ce qui aurait assimilé la discussion à une localisation à l'intérieur d'un contexte international), mais à la poétique de la sensibilité qui attribue aux mots la tâche d'exprimer avec un maximum de raffinement la subtilité des émotions provoquées par la perception de la réalité. Van den Bergh oppose à cela un amour robuste de la vie (Pan revient assez souvent dans ses poèmes) et c'est ce choix vitaliste, un choix philosophique en somme, qui en faisait un exemple attrayant pour nombre de jeunes.

4 L'influence de Van den Bergh fut éphémère, jusque vers 1923, 1924 à peu près, et ceci montre déjà qu'il était avant tout un libérateur et non l'homme qui introduisit une nouvelle idéologie littéraire. Ce rôle fut celui d'un poète tout différent, Martinus Nijhoff (1894-1953). Nijhoff débuta la même année que Paul van Ostaijen, 1916, mais avec un recueil que, s'il faut utiliser des étiquettes, l'on pourrait plutôt appeler symboliste que d'avant-garde. Et malgré tous les glissements que l'on peut constater dans sa poésie, Nijhoff, ne quitte pas la sphère du symbolisme si l'on ne s'en tient pas trop strictement à la définition d'une école (française). Le poète se rapproche le plus de Mallarmé ou de Valéry mais aussi de l'Imagism de T.S. Eliot (s'il avait appartenu à une revue internationale des années vingt, cela aurait été (ommerce). On pourrait dire la même chose de la "théorie poétique " qu'il commence à développer vers 1920. Ce sont ces idées tirées de la poétique "nijhovienne» qui ont eu une influence si forte sur les débats littéraires à la fin des années vingt et au début des années trente que l'on pourrait presque parler d'une dichotomie « Nijhoff », « anti-Nijhoff ».

Les principes que Nijhoff défendait ont une coloration métaphysique. Cette tendance est reprise par l'un des pôles du débat vers 1930, sous une forme si lapidaire et si sclérosée que le mouvement opposé ne pouvait plus que s'exprimer en termes antimétaphysiques privés de nuances. Nijhoff lui-même fut si surpris de toute cette controverse que l'on pourrait se demander s'il pouvait lui-même encore se sentir « nijhovien».

6 Marsman, lui aussi, chef de file désigné des jeunes (Nijhoff était déjà un peu plus âgé et n'était pas non plus enclin à se lancer dans les polémiques) développa sa notion poétique d'«énergie de la forme" sous l'influence de Nijhoff, même si sa poésie abrupte et saccadée, sans mètre et au vers de longueur variable, n'avait par la forme rien de commun avec l'apparence classique qui caractérise les poèmes de Nijhoff. Si nous voulons comprendre les enjeux du débat entre les "métaphysiciens" et les 
«rationalistes » dans les années trente, il nous faudra commencer par bien examiner Nijhoff.

7 Par de nombreux aspects l'« idéologie » littéraire de Nijhoff était apparentée à la thèse de la dépersonnalisation poétique qu'Eliot avait commencé à développer à partir de 1920. Il n'est d'ailleurs pas exclu qu'il se soit agi dans une certaine mesure d'une influence plus ou moins directe mais certainement pas en ce qui concerne les points principaux car Nijhoff avait déjà été préparé à des idées apparentées à celles d'Eliot dès l'école par la lecture de Valéry; ces idées correspondaient à sa recherche et à ses propres convictions.

8 Je ne peux naturellement pas développer une critique approfondie des idées de Nijhoff, je m'en tiendrai au commentaire de quelques citations importantes. Cette remarque tardive résume bien des points importants : «[La finalité de l'art n'est pas une brève prise de conscience] mais la réalisation d'une chose qui dépasse, ne serait-ce que d'un seul centimètre, nos limites ; un fruit qui [...] nous ayant quitté, s'anime lui-même d'une volonté, devient un organisme et ne serait-ce que pour un instant, mène une existence propre, hors de nous, exprimant un peu plus de sens que ce que nous avions pu y mettre.» (1931). Voici les notions clé : "chose", "dépassement de nos limites", « organisme » et « exprimant plus de sens que ce que nous pouvions y mettre ». L'art, et Nijhoff vise presque toujours dans ses critiques l'art littéraire, n'est pas l'expression des profondeurs du moi mais la création d'une forme qui instaure un nouveau contenu, rend une vérité transcendante perceptible. L'expérience personnelle et les émotions forment la base du "moment de création", mais la capacité particulière du poète réside dans ce qu'il abandonne l'aspect strictement personnel et qu'il atteint par les possibilités que procure la langue, une zone située en dehors de nos limites.

Que ceci soit, à la lettre, un point de vue méta-physique, personne ne le niera. « Notre jeune poésie [tend à] l'expression d'une réalité supérieure. » (1925) ; « c'est précisément cette réalité extérieure, cette inhumanité qui dépasse l'humain donne le "divin que l'on attribue à la création poétique, l'« étrange » qui est le propre de tout art. » (1921)

10 Ma méthode de citation à l'emporte-pièce est, je le conçois, dangereuse. Le point de vue de Nijhoff semble vague et péremptoire à la fois si l'on est pas au courant de l'effet objectif qu'ont chez lui ces sortes d'observation et du caractère exact et concret de ses propres poèmes. Mon objectif n'est pas de décrire les conceptions littéraires de Nijhoff dans toutes leurs nuances mais d'en dégager le noyau métaphysique ce qui provoque un effet de grossissement. Je me justifierai de cette simplification d'un point de vue délicat en invoquant le fait que l'influence des conceptions de Nijhoff sur les plus jeunes repose sur un processus de simplification comparable. "Forme créatrice ", un terme que Nijhoff a très souvent employé, depuis 1920 déjà, «la chose dégagée du poète » - voilà, avec des nuances telles que « énergie de la forme » et "impénétrabilité cosmique du poème ", les expressions courantes en poésie dans les années vingt, cellesci se sont même fixées vers 1930 en schibboleth. Ce sont justement ces sortes de définitions du caractère particulier du poème (celui-ci reçoit d'ailleurs bien plus d'attention que le roman par exemple) qui devinrent pour plus d'un esprit rationnel une source de scandale. C'est ainsi que le caractère métaphysique que Nijhoff attribue à la poésie devint le point de départ des polémiques qui ont dominé la littérature néerlandaise entre 1929 et 1935. Le poème, déclare Menno ter Braak (1902-1940) avec un certain goût pour le raisonnement, n'est pas " une chose " « en dehors » ou " audessus » de la personnalité mais l'expression directe de la personnalité elle-même qui 
ne peut être particulière qu'en ce qu'elle exprime l'organisation subtile des moyens linguistiques. Ce ne sont ni "l'impersonnalité » ni une "personnalité extérieure ", « supérieure » ni la « dépersonnalisation » qui caractérisent la bonne poésie mais plutôt la capacité d'exprimer une personnalité marquante. Dans la chaleur du combat on oublia souvent que ce dernier mot comprenait deux notions, celle de "caractère individuel » et celle de « représentant d'un point de vue sans détour ».

11 Il n'est pas étonnant que Nijhoff lui-même se soit tu au cours des discussions auxquelles ses déclarations avaient donné lieu. Ses points de vue avaient disparu derrière le vocabulaire usé de ses adversaires. Chaque poète, chaque lecteur acceptait sa conception de la poésie, mais elle n'était pas en mesure de leur procurer des normes de qualité et c'est exactement ce que le jeune poète D.A.M. Binnendijk (1902-1984) en fit lorsqu'il assura à ses lecteurs dans l'introduction de son anthologie Prisma, que sa sélection dépendait de la mesure dans laquelle le poète a écrit des poèmes qui sont comme « des rameaux détachés de leur origine». En pratique, déclare Ter Braak, cela revenait à une théorie poétique se contentant d'une forme bien construite, sans qu'elle soit soutenue par une personnalité forte, de la poésie d'épigone donc. La " personnalité » s'opposant à la « forme » devint sa devise et celle de son ami et gourou E. du Perron (1899-1940).

12 Cette polarité se compliqua encore par les confrontations philosophiques qui s'y mêlèrent bien que celles-ci n'aient au premier abord rien eu à voir avec l'opposition « forme » / " personnalité ». Dans les années qui suivirent la Première guerre mondiale un mouvement catholique déjeunes se forma qui pour une moitié reprenait le mouvement d'émancipation du dernier quart du siècle et pour l'autre moitié s'opposait justement à ceux-ci à cause de l'opposition de ces jeunes à l'église. Il est éloquent que ce mouvement de jeunes ne s'était pas manifesté dans le sud catholique pratiquant ni dans la ville ou se trouvait la première université catholique du pays, Nimègue, mais à Utrecht où se trouve le siège de l'archevêque et de l'appareil ecclésiastique. Ces jeunes « intégristes » dirigeaient justement leur « artillerie » contre l'embourgeoisement de l'église auquel avait mené l'assimilation et que la génération précédente avait combattue. Ils plaident un parcours radico-social, sans cependant tourner le dos à l'église ; bien souvent ils se sont apparentés au fascisme.

13 Le point de départ philosophique de ces jeunes, qui s'étaient rassemblés autour de la revue De Gemeenschap, allait de pair avec un esthétisme littéraire déjà présent chez leurs pères (la variante néerlandaise de l'Art nouveau est fortement teintée de catholicisme) ; ils avaient néanmoins intégré quelques éléments du renouveau de l'après guerre. Un peu d'expressionnisme, un peu de futurisme, mais cela reste superficiel. Dans les termes de Nijhoff: ils étaient restés accrochés au concept de la forme comme « enveloppe transparente du contenu ». La puissance «créatrice » de la forme capable d'évoquer une nouvelle vérité transcendante ne fait pas partie de leur conception de la littérature. L'aspect métaphysique de la littérature, ils le localisaient dans la phase qui précède la création, la doctrine de l'église en fait, et non à la fin du processus de création. (Je ne peux m'occuper ici des nuances dans les points de vue).

L'accent mis par ces catholiques sur la forme, point de vue qui avait toujours distingué les protestants des catholiques aux Pays-Bas (le rituel s'opposant à l'expérience intime) les rapprocha en apparence des jeunes non-catholiques adeptes des idées de Nijhoff, ils reprenaient tout particulièrement la préférence marquée pour la forme et seulement en parole le rôle oraculaire du poète. La revue des "païens » ou plus exactement des 
« indifférents », De Vrije Bladen était quant aux travaux publiés presque interchangeable avec De Gemeenschap. (Soit dit en passant, les protestants, qui avaient leur propre revue, n'ont joué aucun rôle dans la discussion littéraire de l'époque, ils vivaient isolés et n'avaient que des relations très superficielles avec les autres jeunes). Le rédacteur de Gemeenschap, Jan Engelman (1900-1972) a, par exemple, collaboré à De Vrije Bladen tandis que Marsman, directeur de la revue était tout à fait à l'aise dans la revue catholique (il a même un moment envisagé une conversion au catholicisme).

C'est contre ce complexe de facteurs que Menno ter Braak s'est élevé, contre Nijhoff pour ses prétentions de prophète que d'ailleurs il avait défendues dans sa jeunesse, contre les suiveurs de Nijhoff et leur culte de la forme vide qui menait bien évidemment à une défense de la poésie des épigones et contre les catholiques à cause de leur conceptions figées. À tous ceux-là, en connaisseur et admirateur de Nietzsche il opposait le concept de la (forte) personnalité. Dans les profondeurs de la psychologie, la «théologie de l'expérience » du protestant a pu peut-être jouer un rôle par opposition au ritualisme catholique. Mais réduire la dispute littéraire des jeunes à une sorte d'avatar sécularisé des anciennes guerres de religion mènerait sans doute à une construction quelque peu forcée. Il est cependant évident que Ter Braak a combattu ses ennemis philosophiques de la force de ses arguments mais qu'il n'a pas analysé les idées de Nijhoff avec beaucoup de profondeur (s'il l'avait fait il y aurait découvert des éléments de la philosophie de Nietzsche!) et n'a dirigé ses flèches que contre une version fossilisée de ces idées.

Pour Ter Braak je présenterai de même une paraphrase de ses points de vue et quelques citations qui éclaireront son double combat. Je ne me limiterai pas à l'expression polémique car celle-ci obscurcit chez lui aussi les couches profondes de sa réflexion. Le conflit est souvent, par les antagonistes eux-mêmes, déjà résumé comme une opposition entre la "forme » et le "personnage », une simplification volontaire de la langue allant de pair avec une simplification des enjeux. Mais pourquoi Ter Braak s'oppose-t-il avec tant de fermeté au "culte de la forme" au nom de la "personnalité »? Il s'est exprimé sur la question dès ses plus jeunes années, il n'avait que 24 ans, à une époque ou il ne s'opposait pas encore aux conceptions poétiques de Nijhoff, dans ses essais sur le cinéma il en avait même développé une variante. En résumé son attitude ambivalente vis-à-vis de la forme revient à ceci: pour se manifester la personnalité à besoin de la forme et c'est ce qui la perd. La forme est une nécessité mais elle signe en même temps l'arrêt de mort de la personnalité. L'« individualité » [variante de la personnalité] est résistance, résistance au figé, à l'acceptation sans réflexion, résistance à l'« impersonnel ", une telle conception aurait très bien pu recevoir l'agrément de quelqu'un comme Nijhoff. Mais les conséquences de ce point de vue, tel que Ter Braak l'élaborera à partir de 1929, doivent, pour un Nijhoff, reposer sur un malentendu bizarre. La forme en tant que «mort » de la personnalité, ce n'est pas cela qui a pu lui faire difficulté mais qu'il ne se soit agi que de cela était pour lui, poète dans l'âme, (Ter Braak n'a jamais écrit de poésie) nier la nature véritable de la poésie. La forme produit, disait-il littéralement, un nouveau contenu, elle est la source d'une vie nouvelle qui surpasse l'individu (on pense tout de suite au «si le grain ne meurt» de la Bible). L'admirateur de la forme, pensait par contre Ter Braak, s'abandonne à la séduction de la mort. La personnalité se doit de se détacher de la forme et de recommencer sans cesse tel un Sisyphe. 
L'opposition apparaissant sous cet aspect est plus fructueuse, sûrement dans un débat littéraire, que la question de savoir si la poésie est « un rameau détaché de son origine et de son auteur, un organisme naturel, une fleur » (comme le prétendait Binnendijk) ou bien si elle est l'expression directe d'une "personne » qui ne fait usage que d'une forme déterminée parce qu'elle est la plus efficace pour l'expression d'un point de vue (comme Ter Braak l'affirmait avec plus ou moins de mauvaise humeur). Il va de soi, dit Ter Braak, que « le poète se doit d'être sensible à la valeur émotionnelle de la langue »mais cela n'avait rien à voir avec la question centrale du débat sur la puissance métaphysique de la poésie, Ter Braak avait besoin de cette simplification brutale et de cette restriction du terrain pour mettre ses principes philosophiques en évidence; il laissait de côté le caractère spécifique de l'art (la poésie en particulier) qui caractérisait la prise de position de Nijhoff ou le ridiculisait (« le Grand Poète n'est [en dehors de son œuvre] qu'un petit prolo ») parce que dans le cadre de la confrontation tragique entre la vie et la mort qu'était pour lui l'art, ni plus ni moins que pour Nijhoff, il faisait le choix vital du principe de vie qui résidait à ses yeux dans la « personnalité ». Une bonne partie des critiques que Ter Braak écrivit pendant la première moitié des années trente n'est que la défense d'écrivains dont l'œuvre exprime aussi directement que possible leur conception de la vie. Dans le cas contraire ils se voient "démasqués " (le terme nietzschéen est éloquent), il fallait rechercher leur "deuxième visage ", "l'homme derrière l'œuvre ». Dans les cas extrêmes Ter Braak ne voit dans la poésie qu'un incident dans la biographie de l'auteur.

Le rôle important qu'a joué l'expression polémique de Ter Braak dans la discussion littéraire des années trente, non pas parce qu'il rencontrait l'unanimité mais parce que sa polémique était si virulante qu'elle entrainnait tout le monde dans le même sens, a fait disparaître le caractère spécifique de la littérature (et avec lui la métaphysique nijhovienne) et la discussion se déplaça presque intégralement vers le heurt des conceptions philosophiques et morales. Ce n'est pas pour rien que la revue Forum, née d'une attaque de Ter Braak contre Binnendijk et de l'alliance entre Ter Braak et Du Perron qui s'en était suivie, vit le jour en 1932 : le moment où la menace d'une victoire du national socialisme va occuper le devant de la scène. La suprématie de la critique et de la littérature philosophique est à l'époque plus facile à défendre qu'au début des années vingt. Ces circonstances révèlent que la lutte de Ter Braak (et de Du Perron, mais j'ai choisi l'exemple de Ter Braak) contre les « esthètes » est la continuation de la lutte contre «l'art pour l'art» avec laquelle le point de vue de Nijhoff n'avait strictement rien à voir. Cette prédominance d'une approche philosophique et morale qui signifiait pour Ter Braak le rejet de l'idéologie établie et anti-individualiste, a dominé toutes les années trente jusqu'à ce qu'une nouvelle génération s'interroge non pas sur le choix de Ter Braak pour le «politicien sans parti », qui signifiait finalement un engagement sans équivoque pour la démocratie occidentale, mais sur les limites de sa conception rationnelle des valeurs et du pouvoir de la littérature.

19 Vers la fin des années trente peu d'écrivains néerlandais doutaient de la menace pour leur existence que représentait le courant autoritaire mais un sentiment d'impuissance s'était emparé des intellectuels. Un petit nombre d'écrivains choisit l'engagement (à gauche), la majorité se détourna par contre du débat public et chercha dans l'art une protection qu'ils ne pouvaient trouver nulle part ailleurs. On a parlé de l'« esthétique du bonheur modeste ", mais ce genre de terminologie éveille trop l'impression d'une prise de position claire. Le découragement était en effet le mobile principal de ce 
retrait. De même personne ne pensait plus ni à la "personnalité" ni à la «forme créatrice " jusqu'à ce qu'un groupe de nouveaux auteurs, jeunes moins par l'âge que par leur génération littéraire, brise le silence avec la revue Criterium qui ne commença de paraitre que quelques mois avant l'invasion de 1940.

Le principal porte-parole de [Poésie et métaphysique], Cola Debrot (1902-1981) n'était pas originaire des Pays-Bas, il était né et avait passé sa jeunesse à Curaçao, l'île principale des Antilles néerlandaises. Sa famille a vécu dans les Caraïbes depuis le XVIII ${ }^{e}$ siècle. Ses premiers ancêtres connus du côté paternel étaient des Franco-Suisses protestants alliés à des Néerlandais, et du côté maternel des (catholiques) franco-haïtiens et des vénézuéliens. Cela situait Debrot dans la frange d'un monde culturel néerlandais, mais avec une forte coloration espagnole et française. À l'école où Debrot avait fait ses études il n'y avait pas seulement une section néerlandaise mais aussi une section espagnole (pour les Colombiens les Vénézuéliens etc.). À 14 ans Cola se rendit aux PaysBas car aux Antilles il était impossible de poursuivre des études secondaires et moins encore des études universitaires. Il est ensuite resté trente ans en Europe, faisant tout au plus quelques séjours d'une année environ dans sa patrie et un séjour de six mois aux États-Unis. Il habitait les Pays-Bas où il mena à bien à la fois (ce qui est typiquement caraïbe) des études de droit et de médecine, il vécut également trois ans à Paris où il se lia d'amitié avec Louis-Ferdinand Céline qui venait d'entreprendre l'écriture de son roman Voyage au bout de la nuit.

21 Il n'est guère étonnant que Debrot soit resté un peu étranger à la culture néerlandaise : la langue elle-même était une deuxième langue, sa langue habituelle étant le papiamentu, une langue créole d'influence espagnole. La culture espagnole que Debrot connaissait tout aussi bien (très jeune il avait lu Del Sentimiento Tragico de Miguel de Unamo, la poésie de Sainte Thérèse et de Saint Jean) était pratiquement ignorée aux Pays-Bas. L'auteur Debrot fit des débuts tardifs : sa grande nouvelle Ma sœur la négresse, parue également en français (dans le recueil Nouvelles néerlandaises, Paris 1965), ne fut publiée qu'en 1934. Elle parut dans la revue Forum de Du Perron et Ter Braak qui devinrent ses amis, tout comme Nijhoff, Engelman et Marsman qu'il connaissait déjà. Voilà les deux partis réunis! Cela ne posait d'ailleurs de problème à personne car, tout d'abord, Debrot n'avait pas participé à la discussion sur la "forme » et la " personne ", étranger qu'il était à ce genre de question et bien qu'ayant pris conscience de ses propres objectifs littéraires proches de ceux de Nijhoff grâce aux articles fondamentaux de 1924 et 1925 ; et ensuite, originaire lui-même d'une patrie moins divisée en castes de toutes sortes que la société néerlandaise, il était habitué à la collaboration de familles de pensée et de groupes sociaux opposés.

Debrot réunissait en lui les deux pôles Nijhoff et Ter Braak et cela lui était possible grâce à une vision différente de celle de l'auteur néerlandais moyen. C'est ce qui apparut lorsque, à 38 ans, il fit sa profession de foi dans Criterium. On y compris pas grand-chose, mais ce n'était pas facile car Debrot gardait sa pensée dans l'ombre et essayait de convaincre ses lecteurs en faisant usage des termes de l'ancien débat néerlandais. Une tactique peut-être compréhensible mais qui ne rendait pas les positions de Debrot plus claires.

23 «Oppositions », tel était le titre du premier essai de Debrot dans Criterium et c'était en effet de cela qu'il s'agissait. Non pas à la manière de l'historien de la littérature qui aborde les positions des opposants par la description, mais à partir de l'idée que «les oppositions [sont] le reflet des contradictions tragiques dans l'homme ». Bref, bien plus 
brièvement que ce que Debrot avait fait dans son premier essai programmatique et dans le suivant qui est une réponse aux objections que les critiques (surtout catholiques) avaient apportées à son raisonnement: l'opposition entre une quête " romantique " (mettant en avant le penchant métaphysique) et le côté " rationnel » (visant à la compréhension d'une réalité donnée) est un élément inéluctable de la vie psychique de chaque individu à part entière. La tâche de l'artiste n'est pas d'adoucir ou d'anéantir cette contradiction qui est inhérente au conflit inévitable entre le désir de vivre et l'angoisse de mort. L'objectif de l'artiste est justement de "mettre sous tension » deux tendances intérieures nécessaires chez l'homme (on retrouve un écho évident de Unamuno). Debrot appelle le courant qui se consacre à la confrontation de ces contradictions « Rationalisme romantique » et ses propres poésies et histoires, ceci entre parenthèses, présentent en effet une combinaison permanente d'« ici-bas » et de " céleste ", de vie et de mort, d'obscurité et de lumière, de bien et de mal, d'espoir et de désespoir.

24 À partir de cette position visant l'union (l'un et l'autre) au lieu de l'alternative polémique (l'un ou l'autre), Debrot voit dans le comportement de Ter Braak et Du Perron une action de purification (la tendance métaphysique l'avait emporté dans le monde de la poésie néerlandaise), mais: «nous espérons échapper à l'absence de nuance à laquelle Forum avait dû se déterminer en réaction au radicalisme des autres ».

D'une certaine manière Debrot se rallie donc au désir de transcendance de Nijhoff mais en même temps et avec autant de conviction il défend le droit de Ter Braak de rappeler à l'ordre les « esthètes » qui avaient placé le domaine métaphysique hors de la réalité de l'existence si bien que presque tout le monde interpréta son manifeste-programme comme une conséquence de l'analyse des rédacteurs de Forum tandis qu'il s'agissait plutôt d'un plaidoyer en faveur d'une « métaphysique épurée ». Ter Braak, lui non plus, ne réagit pratiquement pas à cette défense des deux partis en même temps de Debrot parce qu'il n'y voyait qu'une approbation. De plus Ter Braak avait bien sûr lui aussi évolué vers un point de vue moins simpliste. S'il avait pu lire les derniers commentaires de Debrot sur ses positions, il aurait peut-être considéré les choses d'un autre œil, mais lorsque Debrot, après deux ans de silence, en vint à préciser sa conception de la littérature (il n'avait pendant cette période écrit que des nouvelles et des essais sur la danse et la peinture) Ter Braak était déjà mort. À 38 ans, le 14 mai 1940, jour de la capitulation des Pays-Bas, il s'était suicidé. Simultanément son ami Du Perron succombait à une crise cardiaque. Marsman se noyait quelques mois plus tard en essayant d'atteindre les côtes anglaises: une génération littéraire disparaissait d'un seul coup. Il n'est pas impossible que Debrot, à la mort de ses amis, se soit cru obligé de reprendre le flambeau.

26 Ce n'est qu'en mai 1942 que Debrot reprend le fil de la polémique de Criterium, in extremis car quelques mois plus tard la revue cessa de paraitre. L'envahisseur interdisait toute activité culturelle publique ne répondant pas aux exigences des principes nazis. L'article dans lequel Debrot poursuit sa réflexion est consacré à Martinus Nijhoff, le fait est déjà éloquent en soi.

Sans reprendre explicitement son programme de 1940, Debrot développe dans cet essai, qu'il baptise "l'heure de l'accomplissement", l'unité dans la polarité qu'il avait défendue deux ans auparavant. Phrase-clé dont le vocabulaire trahit déjà la continuité par rapport à l'article programme: "Ce que ce poète recherchait [...] c'est sa métaphysique dont la réalité sera le symbole.» Dans le contexte cette affirmation est 
moins obscure qu'il n'y paraît à première vue; je donnerai donc le commentaire l'accompagnant dans le texte lui-même de "l'heure de l'accomplissement », mais qu'on retrouve dans de nombreux essais de cette période et même dans les romans et les nouvelles.

Nijhoff, écrit Debrot, balançait dans ses premiers poèmes entre le désespoir le plus profond et l'acceptation la plus totale de la vie. Mais à présent ils constituent en quelque sorte les deux pôles d'un même aimant : c'est « l'accomplissement » dont il est question. Au départ Nijhoff était à la recherche de la lumière en dehors de soi et hors de la réalité tangible, c'est-à-dire dans une capacité particulière, au delà de l'individu, de la langue à laquelle le poète se confie sans restriction aucune. Ce pouvoir de la langue, de la "forme ", est, selon Debrot, irréfutable mais n'avoir d'attention que pour cela et pour rien d'autre, c'est ouvrir la porte à une esthétique qui se perd dans des élucubrations sans âme et poursuit l'art et l'humanité hors de la réalité (la métaphysique « élyséenne » des jeunes catholiques). C'est justement là que le plaidoyer de Ter Braak pour la personnalité passionnelle a un effet purificateur en remettant le poète sur terre, dans la réalité de la responsabilité personnelle.

Nijhoff s'est libéré des « Geister die er aufgerufen » dès qu'il s'est mis à chercher le sens de l'art dans une expérience plus intense de la réalité. Ne plus voir les choses comme des éléments qui constituent le décor de la vie personnelle, mais les vivre comme porteuses d'une existence autonome, ce qui est aussi "transcendant", "métaphysique", mais on ne perd pas la réalité de vue. La conscience intense de l'«être ", de l'existant (c'est ainsi que s'exprimait Debrot depuis de nombreuses années) appelle en même temps une angoisse de l'acceptation de la réalité. C'est l'intensité de cette perception de la réalité qui permet à l'artiste de transcender l'accidentel, c'est là que réside sa métaphysique.

30 En relisant la citation que nous venons de faire de Nijhoff, « la métaphysique dont la réalité est le symbole », tout est devenu, je l'espère, parfaitement clair.

31 Knuvelder, l'adversaire catholique le plus courtois et le plus réfléchi deux plus tôt, a immédiatement compris que dans le long essai sur Nijhoff, Debrot reprenait le fil de l'ancienne discussion, et dans des termes moins vagues et généraux, mais mis en évidence grâce à un exemple accessible. Il examine le nouvel essai de Debrot à la loupe et pour la première fois le choix qui avait été l'enjeu de la discussion est enfin clair. Knuvelder objecte que Debrot ne «quittait pas son complexio oppositorum » et ne parvient pas à la «concordantia diacordantium ». L'œuvre d'art, affirme-t-il, exprime cependant la "concordantia», l'harmonie (qui pour Knuvelder est bien sûr incluse dans la doctrine de l'église qui réconcilie l'homme, et l'artiste, avec la vie).

Le raisonnement de Knuvelder tombe bien à propos pour Debrot, il peut maintenant franchir une étape décisive. Si Knuvelder, dit-il, seulement voulait exprimer que l'artiste place le "complexio oppositorum » dans une forme fermée et crée par là une certaine harmonie, il ne le contredira pas, mais Knuvelder fait plus que cela: il place l'harmonie, la réconciliation des contraires avant l'acte artistique. Pour écarter tout malentendu y compris celui d'un accord qui reposerait sur une méprise, Debrot introduit une nouvelle paire de termes: «cosmique» par opposition à « eschatologique» (les connaisseurs reconnaîtront ici Unamuno, qu'il cite cette fois expressis verbis). Pour exprimer clairement ce qu'il veut dire, Debrot retourne au passé lointain du XVII ${ }^{e}$ siècle, en adoucissant par là la controverse polémique, mais accentuant en même temps la continuité historique de la dichotomie. 

rythme soutenu), leurs représentants n'éprouvaient aucunement le besoin de se rapprocher de leur prédécesseurs. Bien au contraire, ils firent des poètes de Criterium, écrivains de sonnets à la thématique souvent modeste, l'arrière-plan grisaille sur lequel se détachait vivement, par contraste, leur propre poésie qui ne dédaignait pas les effets. Mais la pensée de Debrot, qui ne faisait nullement partie de la "poésie de Criterium » dans le sens péjoratif du terme, ne rencontra aucun intérêt de la part des jeunes. C'était un nouvel élan littéraire et le fait que celui-ci ait pu avoir des affinités avec les idées 
que Debrot avaient lancées en 1940-1942 aurait représenté, si cela avait été remarqué, plutôt un obstacle à surmonter qu'un soutien. L'œuvre du plus grand des nouveaux poètes, Lucebert (1924-1994), présente indéniablement aussi bien un lien profond avec la réalité, et l'actualité même, qu'une aspiration métaphysique, mais le débat sur la "métaphysique» et le "rationalisme», sur les artistes " eschatologiques » et le « cosmique » a vécu. Le loup et l'agneau sont en effet couchés côte à côte, dans la tombe de l'histoire littéraire.

\section{RÉSUMÉS}

Le débat littéraire aux Pays-Bas, contrairement à ce qui fut le cas en Flandre, s'est tenu à l'écart de la vie intellectuelle du reste de l'Europe. L'opposition entre « l'art pour l'art » et l'engagement a joué un rôle plus profond, de pair avec le conflit traditionnel entre catholicisme et protestantisme, romantisme et rationalisme, métaphysique et réalisme. Les personnages principaux de ce débat : Slauerhoff, Théo van Doesburg mais surtout Nijhoff, Marsman, Ter Braak et enfin Cola Debrot qui fut la synthèse des deux pôles (Nijhoff et Ter Braak).

De Nederlandse literaire discussie heeft zieh, in tegenstelling tot de Vlaamse, afzijdig gehouden van de rest van Europa. Het contrast tussen «l'art pour l'art » en maatschappelijk engagement heeft in het Noorden een längere doorwerking gehad, dit samen met de traditionele strijd tussen katholicisme en protestantisme, romantisme en rationalisme, metafysiek en realisme. De hoofdpersonnages van dit debat: Slauerhoff, Theo van Doesburg maar vooral Martinus Nijhoff, Marsman, Ter Braak en uiteindelijk Cola Debrot dit de twee belangrijkste polen, Nijhoff en Ter Braak, in zieh verenigde. 